\title{
Attualità dalla Svizzera italiana
}

Nicola Gianinazzi

In occasione del giubileo per il $40^{\circ}$ dell'ASP inseriamo questo breve paragrafo storico.

In quest'ultimo decennio l'ASP ha anche visto nascere l'esperienza della sua Sezione della Svizzera italiana: essa nacque sullintuizione del suo allora direttore Emil Stutz e del sottoscritto con lobiettivo di rivitalizzare l'Associazione sul suo versante italofono. Già la prima riunione «costitutiva» - nel 2010 - vide la forte partecipazione di una ventina di membri (quasi tutti gli iscritti di lingua italiana) e la simpatica cena che seguì la riunione sancì definitivamente l'inizio ufficiale di questa esperienza.

La Sezione portò parecchi nuovi membri verso l'ASP - tra cui diversi giovani ma non solo - un membro collettivo e nuovamente un suo delegato nella Commissione consultiva cantonale per le professioni di psicologo e psicoterapeuta.

Ora le sue attività di consulenza, di rete, di «animazione formativa» e «mediazione culturale» vengono da più di un anno portate avanti nella forma più snella di una delegazione per la Svizzera italiana.

\section{Dalla Svizzera italiana}

Forniamo regolarmente consulenze individuali a colleghi psicologi e psicoterapeuti, nonché occasionalmente a Istituti di formazione svizzeri ed esteri e ad altre Istituzioni private e pubbliche.

Per quanto riguarda invece il lavoro intra-associativo esso consiste soprattutto di mediazione tra realtà regionale e nazionale, di consulenza e di revisione e traduzione di testi informativi o più prettamente tecnici.

Si continua a collaborare con profitto con l'Istituto Ricerche di Gruppo per lorganizzazione della formazione continua, così da poter garantire sempre una vasta scelta di corsi di qualità. I corsi possono così essere scelti nellambito del Curriculum di formazione postgraduale secondo il concetto ASP Integral.

Inoltre dal 2018 il sottoscritto fa parte della redazione di Psychotherapie-Wissenschaft - la Svizzera italiana e il panorama italiano saranno più presenti e visibili nella nostra rivista tematica.

Continua il lavoro di contatto con docenti universitari di varie università e facoltà ticinesi. Questa interessante attività di "public relations» è utile e necessaria per arricchire sia le proposte formative, sia i contributi delle nostre riviste associative. In quest'ultimo ambito si approfon- disce pure la collaborazione redazionale con la rivista Psicoterapia e scienze umane, di cui è co-direttore Paolo Migone.

\section{Panorama psicoterapeutico ticinese e tran- sfrontaliere}

In Ticino - nel contesto di una collaborazione internazionale che coinvolge diversi membri ASP, ricercatori accademici e altri partners ancora - è nato un sito dedicato alla ricerca nell'ambito sanitario-medico, della psicoterapia, della psicologia e delle scienze umane in genere. La sua Mission consiste nell'individuazione, la progettazione e l'implementazione di progetti di ricerca che esplorino tematiche psicologiche e psico-sociali importanti per i cittadini residenti nel Canton Ticino. I nostri progetti non hanno un fine esclusivamente conoscitivo, anche se come ricercatori per noi la conoscenza ha un valore fondamentale. Essi mirano ad approfondire tematiche e fenomeni psicologici e psico-sociali che siano particolarmente rilevanti per i cittadini residenti in Canton Ticino. Infatti, nello sviluppo di tutti i nostri progetti poniamo particolare attenzione alle peculiarità, alle specificità ma sopratutto alle esigenze del nostro territorio.

La Mission di «Psychology Research Ticino» non è la mera osservazione e catalogazione dei fenomeni psicologici e sociali che si sviluppano in Ticino, ma vogliamo utilizzare le conoscenze emerse dai nostri studi per proporre e costruire programmi di educazione, promozione e supporto alla salute che siano realmente implementabili sul territorio e che siano utili e funzionali a migliorare la qualità di vita dei cittadini residenti nel nostro Cantone. La Mission di «Psychology Research Ticino» in poche parole:

- Promuovere la ricerca scientifica in ambito psicologico e psico-sociale sul territorio ticinese

- Coinvolgere attivamente i residenti in Canton Ticino nei progetti di ricerca promossi da «Psychology Research Ticino» promuovendo un clima di collaborazione, partecipazione attiva e responsabilità individuale rispetto alle tematiche relative alla salute

- Condividere con i residenti del Canton Ticino i risultati ottenuti dai progetti di ricerca promossi da «Psychology Research Ticino» con lo scopo sia di «educare» i cittadini rispetto a tematiche di salute e benessere importanti per la qualità di vita individuale

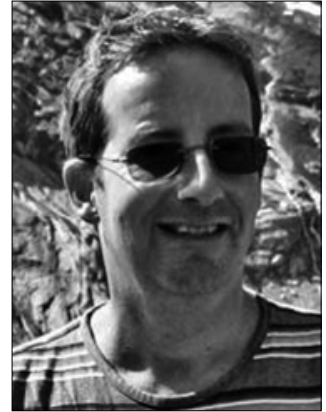


- Divulgare ai non professionisti della salute, utilizzando un linguaggio adatto a tutti ma un profondo rigore scientifico, le principali novità riguardanti la psicologia e la psicoterapia

- Formare i professionisti della salute attivi sul territorio ticinese agli aspetti inerenti la nostra attività di ricerca

Campi di applicazione principali per «Psychology Research Ticino»:

- Valutazione e promozione della salute mentale e del benessere psicologico nella popolazione residente in Canton Ticino
- Studio e prevenzione dei comportamenti a rischio negli adolescenti e giovani adulti residenti in Canton Ticino

- Valutazione delle problematiche e delle difficoltà di adattamento negli adolescenti e nei giovani adulti residenti in Canton Ticino

- Valutazione delle problematiche e delle difficoltà di adattamento delle famiglie residenti in Canton Ticino

Link del sito di «Psychology Research Ticino»: https://www.psychologyresearchticino.ch/Home

Nicola Gianinazzi è membro di comitato e delegato per la Svizzera italiana.

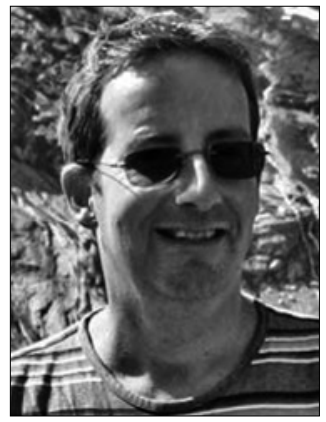

\section{Nouvelles de la Suisse italienne}

Nicola Gianinazzi

À l'occasion du 40e anniversaire de l'ASP, nous publions ce petit paragraphe historique.

Dans la dernière décennie, l'ASP a vu naître sa section Suisse italienne, née de l'initiative de son directeur de l'époque, Emil Stutz, et de moi-même, dans le but de redynamiser le versant italophone de l'Association. Dès la première réunion de "constitution ", en 2010, la participation fut forte avec une vingtaine de membres (soit la quasi-totalité des adhérents de langue italienne) et le dîner sympathique qui s'ensuivit marqua définitivement le lancement officiel de l'expérience.

La Section a amené beaucoup de nouveaux membres à l'ASP - un certain nombre de jeunes notamment, mais aussi de moins jeunes - un membre collectif et, récemment, un de ses délégués siège à la Commission consultative cantonale des professions de la psychologie et de la psychothérapie.

Aujourd'hui, ses activités de conseil, de mise en réseau, dorganisation de formations et de médiation culturelle sont mises en avant depuis plus d'un an sous la forme plus concise d'une délégation Suisse italienne.

\section{Depuis la Suisse italienne}

Nous fournissons régulièrement des conseils personnalisés à des confrères psychologues et psychothérapeutes et, occasionnellement, à des instituts de formation suisses et étrangers et à d'autres institutions privées et publiques.

Par ailleurs, au sein de l'association, le travail consiste surtout en une médiation entre les entités régionale et nationale, en conseils, en révisions et en traductions de textes d'information ou à caractère technique.

Nous poursuivons notre collaboration fructueuse avec l'Istituto Ricerche di Gruppo (IRG) pour lorganisation de la formation continue, de manière à pouvoir garantir le maintien d'un vaste éventail de cours de qualité. Les cours peuvent ainsi être choisis dans le cadre du parcours de formation postgrade selon le concept ASP Integral.

En outre, depuis 2018, je suis entré dans la rédaction de Sciences psychothérapeutiques, la Suisse italienne et le contexte italien vont ainsi gagner en présence et en visibilité dans notre revue thématique.

Les contacts avec des professeurs de différentes universités et facultés du Tessin se poursuivent. Cette activité stimulante de relations publiques est utile et nécessaire pour enrichir les propositions de formation et les contributions aux revues de l'association. Dans ce cadre, la collaboration rédactionnelle avec la revue Psicoterapia e scienze umane, codirigée par Paolo Migone, est également renforcée. 